\title{
Contribuição ao estudo alelopático de Erythrina velutina Willd., Fabaceae
}

\author{
Caroline Centenaro, ${ }^{1}$ Luís G. P. Corrêa, ${ }^{1}$ Marcos J. Karas, ${ }^{1}$ Suzane Virtuoso, ${ }^{2}$ Josiane F. G. \\ Dias, ${ }^{2}$ Obdulio G. Miguel, ${ }^{2}$ Marilis D. Miguel ${ }^{*, 1}$ \\ ${ }^{1}$ Laboratório de Farmacotécnica, Departamento de Farmácia, Universidade Federal do Paraná, 80210-170 \\ Curitiba-PR, Brasil, \\ ${ }^{2}$ Laboratório de Fitoquímica, Departamento de Farmácia, Universidade Federal do Paraná, 80210-170 \\ Curitiba-PR, Brasil
}

\begin{abstract}
RESUMO: Este trabalho utilizou extrato etanólico e respectivas frações de Erythrina velutina em ensaio alelopático com sementes de Lactuca sativa. Observou-se que, com exceção da fração acetato de etila, o extrato etanólico e frações foram capazes de influenciar a germinação e crescimento das sementes de L. sativa.
\end{abstract}

Unitermos: Alelopatia, Lactuca sativa, Erythrina, Fabaceae.

\begin{abstract}
Contribution to the allelopatic study of Erythrina velutina Willd., Fabaceae". This work has used ethanol extract and respective fractions from Erythrina velutina in allelophatic rehearsal with seeds from Lactuca sativa. It was observed that, except for the ethyl acetate fraction, the ethanol extract and fractions has been capable to influence the germination and growth of the seeds from L. sativa.
\end{abstract}

Keywords: Allelopathy, Lactuca sativa, Erythrina, Fabaceae.

\section{INTRODUÇÃO}

As plantas pertencentes ao gênero Erythrina são fontes de alcalóides tetracíclicos do tipo eritrina e possuem em sua composição química flavonóides, cumarinas e saponinas (Cunha et al., 1996; Rabelo et al., 2001; Virtuoso et al., 2005; Corrêa et al., 2008; Sousa et al., 2008). Erythrina velutina Willd., Fabaceae é conhecida como suinã, mulungu, canivete e corticeira (Lorenzi, 2002). Apresenta a sinonímia botânica: Corallodendron velutinum Willd., Erythrina aculeastissima Desf., Erythrina splendida Diels e Chirocalyx velutinus Walp. (Lorenzi, 2002). É utilizada popularmente como sedativo, calmante, sudorífica, emoliente, anestésico local e para acelerar a maturação de abcessos da gengiva (Lorenzi, 2002; Agra et al., 2008; Reyes, 2008). Estudos farmacológicos demonstraram que o extrato de E. velutina possui atividade espasmolítica, curarizante, antimuscarínica e depressora do Sistema Nervoso Central, propriedades compatíveis com as preconizadas pelo uso popular (Reyes, 2008).

O termo alelopatia foi citado pela primeira vez em 1937 pelo alemão Hans Molish, citada por diversos autores ao longo dos anos e atualmente definida pela International Allelopathy Society como processos que envolvem a produção de metabólitos secundários por plantas e microrganismos que influenciam no crescimento e desenvolvimento de sistemas biológicos com efeitos positivos e negativos (Malheiros \& Perez, 2001; Pinto et al., 2002). Sendo assim, aceita-se alelopatia como ciência abrangente, podendo ser utilizada no controle de doenças, insetos e plantas daninhas que acometem plantas medicinais, proporcionando matéria-prima com qualidade para a indústria de fitoterápicos (Dias, 2005). Nesta perspectiva, este trabalho teve como objetivo verificar atividade alelopática de extratos e frações de E. velutina.

\section{MATERIAL E MÉTODOS}

Os solventes e reagentes utilizados apresentaram grau de pureza analítico (Merck $\left.{ }^{\circledR}\right)$. Cascas de Erythrina velutina foram colhidas no Nordeste do Brasil em 2005, a classificação da espécie foi realizada junto à EMBRAPA e encontra-se sob registro $\mathrm{n}^{\circ} .2298$.

O extrato bruto etanólico (EB) foi obtido pela submissão das cascas (secas e estabilizadas) à extração etanólica (etanol 96\%, durante seis horas) em aparelho de Soxhlet e posterior concentração em evaporador rotatório com pressão reduzida à temperatura de 40 ${ }^{\circ} \mathrm{C}$ e $90 \mathrm{rpm}$ até redução a $1 / 5$ de seu volume. O EB foi fracionado em aparelho de Soxhlet modificado (Carvalho, 2001) com solventes em ordem crescente 
Tabela 1. Germinação e crescimento de Lactuca sativa frente ao extrato bruto etanólico e fração hexano de E. velutina.

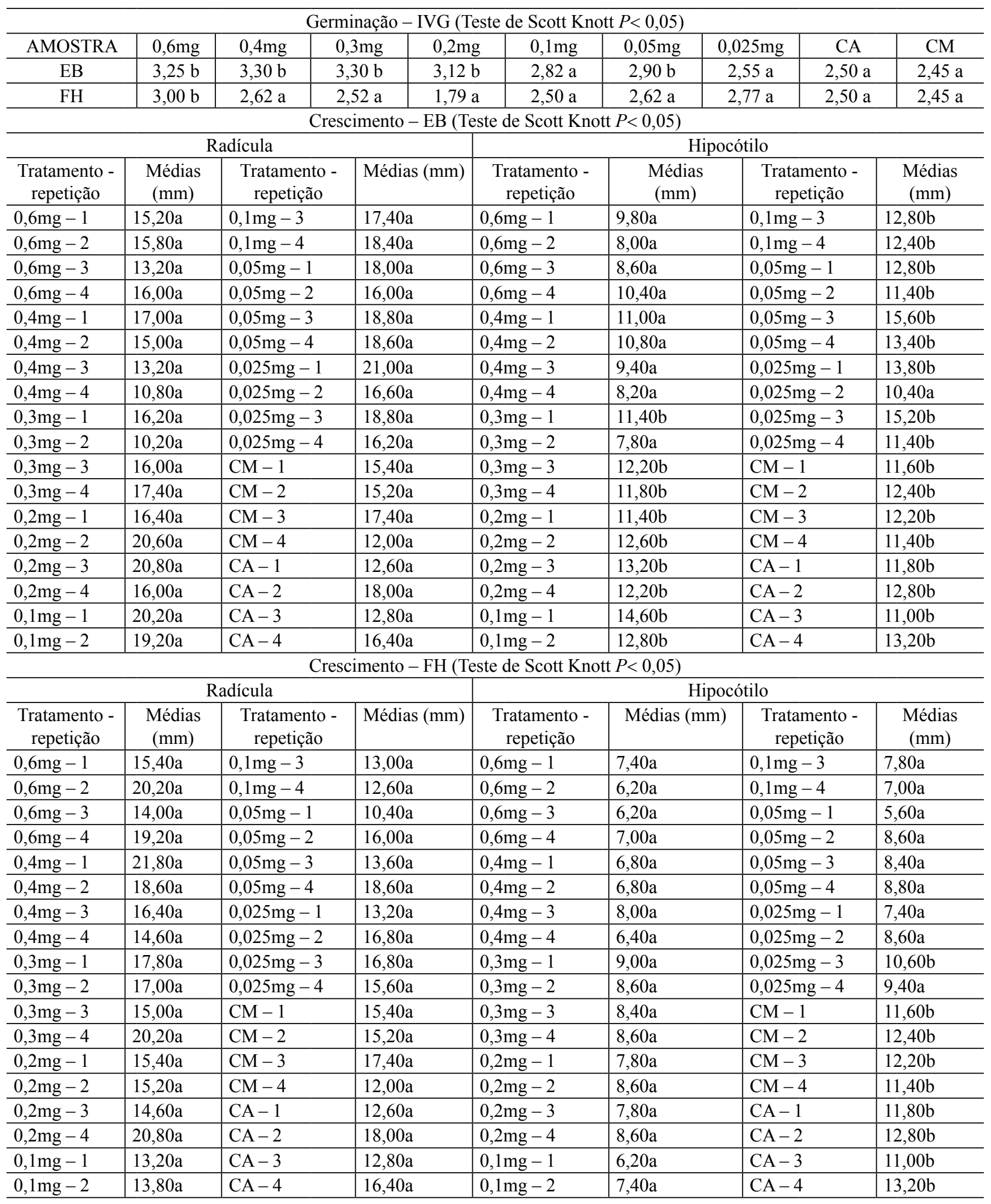

$\mathrm{CA}=$ controle água $\mathrm{CM}=$ controle metanol; $\mathrm{EB}=$ extrato bruto etanólico e $\mathrm{FH}=$ fração hexano.

de polaridade: hexano, clorofórmio e acetato de etila, obtendo-se a fração hexano $(\mathrm{FH})$, fração clorofórmio (FC), fração acetato de etila (FAE) e fração hidroalcóolica remanescente (FHR).

O ensaio alelopático utilizou o extrato bruto etanólico e respectivas frações nas concentrações de 0,6 $\mathrm{mg}, 0,4 \mathrm{mg}, 0,3 \mathrm{mg}, 0,2 \mathrm{mg}, 0,1 \mathrm{mg}, 0,05 \mathrm{mg}$ e $0,25 \mathrm{mg}$ diluídas em $2 \mathrm{~mL}$ de metanol e sementes de Lactuca sativa (Macías et al., 2000; Chon et al., 2005; Dias et al., 2005). 
Tabela 2. Germinação e crescimento de Lactuca sativa frente a fração clorofórmio e fração acetato de etila de E. velutina.

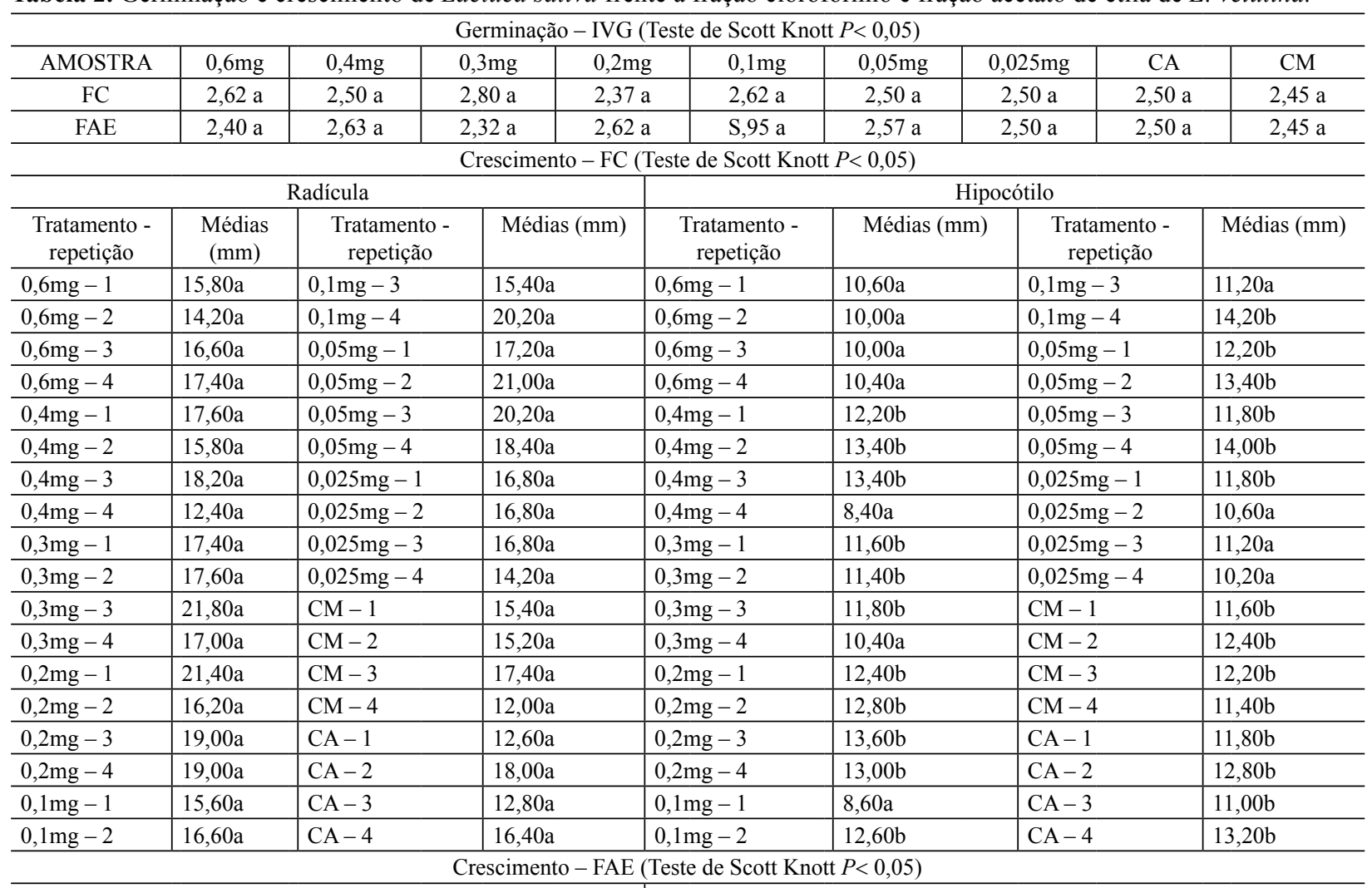

\begin{tabular}{c|l|l|l|l|l|l|l}
\hline \multicolumn{3}{c}{ Radícula } & \multicolumn{1}{c}{ Hipocótilo } \\
\hline $\begin{array}{c}\text { Tratamento - } \\
\text { repetição }\end{array}$ & $\begin{array}{c}\text { Médias } \\
(\mathrm{mm})\end{array}$ & $\begin{array}{c}\text { Tratamento - } \\
\text { repetição }\end{array}$ & Médias (mm) & $\begin{array}{c}\text { Tratamento - } \\
\text { repetição }\end{array}$ & \multicolumn{1}{c}{$\begin{array}{c}\text { Médias } \\
(\mathrm{mm})\end{array}$} & $\begin{array}{c}\text { Tratamento - } \\
\text { repetição }\end{array}$ & \multicolumn{1}{c}{ Médias (mm) } \\
\hline $0,6 \mathrm{mg}-1$ & $16,00 \mathrm{a}$ & $0,1 \mathrm{mg}-3$ & $17,80 \mathrm{a}$ & $0,6 \mathrm{mg}-1$ & $8,60 \mathrm{a}$ & $0,1 \mathrm{mg}-3$ & $12,00 \mathrm{~b}$ \\
\hline $0,6 \mathrm{mg}-2$ & $18,40 \mathrm{a}$ & $0,1 \mathrm{mg}-4$ & $12,20 \mathrm{a}$ & $0,6 \mathrm{mg}-2$ & $11,40 \mathrm{~b}$ & $0,1 \mathrm{mg}-4$ & $8,60 \mathrm{a}$ \\
\hline $0,6 \mathrm{mg}-3$ & $15,00 \mathrm{a}$ & $0,05 \mathrm{mg}-1$ & $17,40 \mathrm{a}$ & $0,6 \mathrm{mg}-3$ & $9,40 \mathrm{a}$ & $0,05 \mathrm{mg}-1$ & $12,80 \mathrm{~b}$ \\
\hline $0,6 \mathrm{mg}-4$ & $18,20 \mathrm{a}$ & $0,05 \mathrm{mg}-2$ & $15,20 \mathrm{a}$ & $0,6 \mathrm{mg}-4$ & $13,20 \mathrm{~b}$ & $0,05 \mathrm{mg}-2$ & $13,00 \mathrm{~b}$ \\
\hline $0,4 \mathrm{mg}-1$ & $16,60 \mathrm{a}$ & $0,05 \mathrm{mg}-3$ & $17,40 \mathrm{a}$ & $0,4 \mathrm{mg}-1$ & $13,40 \mathrm{~b}$ & $0,05 \mathrm{mg}-3$ & $12,80 \mathrm{~b}$ \\
\hline $0,4 \mathrm{mg}-2$ & $18,20 \mathrm{a}$ & $0,05 \mathrm{mg}-4$ & $13,40 \mathrm{a}$ & $0,4 \mathrm{mg}-2$ & $13,00 \mathrm{~b}$ & $0,05 \mathrm{mg}-4$ & $8,80 \mathrm{a}$ \\
\hline $0,4 \mathrm{mg}-3$ & $15,40 \mathrm{a}$ & $0,025 \mathrm{mg}-1$ & $14,40 \mathrm{a}$ & $0,4 \mathrm{mg}-3$ & $10,80 \mathrm{a}$ & $0,025 \mathrm{mg}-1$ & $10,20 \mathrm{a}$ \\
\hline $0,4 \mathrm{mg}-4$ & $10,20 \mathrm{a}$ & $0,025 \mathrm{mg}-2$ & $13,00 \mathrm{a}$ & $0,4 \mathrm{mg}-4$ & $8,40 \mathrm{a}$ & $0,025 \mathrm{mg}-2$ & $9,40 \mathrm{a}$ \\
\hline $0,3 \mathrm{mg}-1$ & $16,40 \mathrm{a}$ & $0,025 \mathrm{mg}-3$ & $15,00 \mathrm{a}$ & $0,3 \mathrm{mg}-1$ & $13,60 \mathrm{~b}$ & $0,025 \mathrm{mg}-3$ & $12,80 \mathrm{~b}$ \\
\hline $0,3 \mathrm{mg}-2$ & $14,40 \mathrm{a}$ & $0,025 \mathrm{mg}-4$ & $17,60 \mathrm{a}$ & $0,3 \mathrm{mg}-2$ & $12,60 \mathrm{~b}$ & $0,025 \mathrm{mg}-4$ & $12,20 \mathrm{~b}$ \\
\hline $0,3 \mathrm{mg}-3$ & $15,00 \mathrm{a}$ & $\mathrm{CM}-1$ & $15,40 \mathrm{a}$ & $0,3 \mathrm{mg}-3$ & $9,60 \mathrm{a}$ & $\mathrm{CM}-1$ & $11,60 \mathrm{~b}$ \\
\hline $0,3 \mathrm{mg}-4$ & $17,60 \mathrm{a}$ & $\mathrm{CM}-2$ & $15,20 \mathrm{a}$ & $0,3 \mathrm{mg}-4$ & $13,20 \mathrm{~b}$ & $\mathrm{CM}-2$ & $12,40 \mathrm{~b}$ \\
\hline $0,2 \mathrm{mg}-1$ & $12,60 \mathrm{a}$ & $\mathrm{CM}-3$ & $17,40 \mathrm{a}$ & $0,2 \mathrm{mg}-1$ & $11,80 \mathrm{~b}$ & $\mathrm{CM}-3$ & $12,20 \mathrm{~b}$ \\
\hline $0,2 \mathrm{mg}-2$ & $16,00 \mathrm{a}$ & $\mathrm{CM}-4$ & $12,00 \mathrm{a}$ & $0,2 \mathrm{mg}-2$ & $9,20 \mathrm{a}$ & $\mathrm{CM}-4$ & $11,40 \mathrm{~b}$ \\
\hline $0,2 \mathrm{mg}-3$ & $15,80 \mathrm{a}$ & $\mathrm{CA}-1$ & $12,60 \mathrm{a}$ & $0,2 \mathrm{mg}-3$ & $12,20 \mathrm{~b}$ & $\mathrm{CA}-1$ & $11,80 \mathrm{~b}$ \\
\hline $0,2 \mathrm{mg}-4$ & $20,00 \mathrm{a}$ & $\mathrm{CA}-2$ & $18,00 \mathrm{a}$ & $0,2 \mathrm{mg}-4$ & $13,60 \mathrm{~b}$ & $\mathrm{CA}-2$ & $12,80 \mathrm{~b}$ \\
\hline $0,1 \mathrm{mg}-1$ & $16,40 \mathrm{a}$ & $\mathrm{CA}-3$ & $12,80 \mathrm{a}$ & $0,1 \mathrm{mg}-1$ & $9,60 \mathrm{a}$ & $\mathrm{CA}-3$ & $11,00 \mathrm{~b}$ \\
\hline $0,1 \mathrm{mg}-2$ & $13,40 \mathrm{a}$ & $\mathrm{CA}-4$ & $16,40 \mathrm{a}$ & $0,1 \mathrm{mg}-2$ & $11,40 \mathrm{~b}$ & $\mathrm{CA}-4$ & $13,20 \mathrm{~b}$ \\
\hline $\mathrm{CA}=$
\end{tabular}

$\mathrm{CA}=$ controle água; $\mathrm{CM}=$ controle metanol; $\mathrm{FC}=$ fração clorofórmio e $\mathrm{FAE}$ = fração acetato de etila.

Para tratamento controle da germinação e do crescimento utilizou-se água destilada e metanol sob as mesmas condições. Para verificação da germinação, procedeu-se leitura diária durante sete dias com retirada das sementes germinadas. As sementes foram consideradas germinadas conforme descrito por De Feo et al. (2002) e Adegas et al. (2003), ou seja, quando se tornou visível a protrusão da radícula através do tegumento. A abertura diária das caixas Gerbox foi realizada em fluxo laminar. Calculou-se o índice da velocidade de germinação segundo Maguire (1962) e os dados obtidos foram submetidos ao Teste de Scott Knott (5\% de probabilidade) (Ferreira, 2000).

Para verificação do crescimento realizou-se ao final do sétimo dia de experimento, leitura do crescimento do hipocótilo e da radícula em papel milimetrado com auxílio de pinça. Os dados obtidos com as leituras de hipocótilo e radícula foram submetidos ao Teste de 
Tabela 3. Germinação e crescimento de Lactuca sativa frente a fração hidroalcóolica remanescente de E. velutina.

\begin{tabular}{|c|c|c|c|c|c|c|c|c|c|c|}
\hline \multicolumn{11}{|c|}{ Germinação - IVG (Teste de Scott Knott $P<0,05$ ) } \\
\hline AMOSTRA & $0,6 \mathrm{mg}$ & $0,4 \mathrm{mg}$ & $0,3 \mathrm{mg}$ & \multicolumn{2}{|c|}{$0,2 \mathrm{mg}$} & $0,1 \mathrm{mg}$ & $0,05 \mathrm{mg}$ & $0,025 \mathrm{mg}$ & CA & $\mathrm{CM}$ \\
\hline FHR & $2,62 \mathrm{a}$ & $2,50 \mathrm{a}$ & $3,25 \mathrm{~b}$ & \multicolumn{2}{|c|}{$2,75 \mathrm{a}$} & $2,87 \mathrm{a}$ & $2,75 \mathrm{a}$ & $2,75 \mathrm{a}$ & $2,50 \mathrm{a}$ & $2,45 \mathrm{a}$ \\
\hline \multicolumn{11}{|c|}{ Crescimento - FHR (Teste de Scott Knott $P<0,05)$} \\
\hline \multicolumn{5}{|c|}{ Radícula } & \multicolumn{6}{|c|}{ Hipocótilo } \\
\hline $\begin{array}{l}\text { Tratamento - } \\
\text { repetição }\end{array}$ & Médias (mm) & $\begin{array}{c}\begin{array}{c}\text { Tratamento - } \\
\text { repetição }\end{array} \\
\end{array}$ & \multicolumn{2}{|c|}{ Médias (mm) } & & $\begin{array}{l}\text { ratamento - } \\
\text { repetição }\end{array}$ & $\begin{array}{l}\text { Médias } \\
(\mathrm{mm})\end{array}$ & \multicolumn{2}{|c|}{\begin{tabular}{c|c}
$\begin{array}{c}\text { Tratamento - } \\
\text { repetição }\end{array}$ \\
\end{tabular}} & $\begin{array}{l}\text { Médias } \\
(\mathrm{mm})\end{array}$ \\
\hline $0,6 \mathrm{mg}-1$ & $17.00 \mathrm{a}$ & $0,1 \mathrm{mg}-3$ & \multicolumn{2}{|c|}{$14,60 \mathrm{a}$} & \multicolumn{2}{|c|}{$0,6 \mathrm{mg}-1$} & $9,20 \mathrm{a}$ & \multicolumn{2}{|c|}{$0,1 \mathrm{mg}-3$} & $10,00 \mathrm{a}$ \\
\hline $0,6 \mathrm{mg}-2$ & $14,80 \mathrm{a}$ & $0,1 \mathrm{mg}-4$ & \multicolumn{2}{|c|}{$16,60 \mathrm{a}$} & \multicolumn{2}{|c|}{$0,6 \mathrm{mg}-2$} & $11,20 \mathrm{~b}$ & \multicolumn{2}{|c|}{$0,1 \mathrm{mg}-4$} & $13,20 \mathrm{~b}$ \\
\hline $0,6 \mathrm{mg}-3$ & $15,40 \mathrm{a}$ & $0,05 \mathrm{mg}-1$ & \multicolumn{2}{|c|}{$15,00 \mathrm{a}$} & \multicolumn{2}{|c|}{$0,6 \mathrm{mg}-3$} & $10,40 \mathrm{a}$ & \multicolumn{2}{|c|}{$0,05 \mathrm{mg}-1$} & $10,20 \mathrm{a}$ \\
\hline $0,6 \mathrm{mg}-4$ & $13,30 \mathrm{a}$ & $0,05 \mathrm{mg}-2$ & 12,60 & & & $g-4$ & $11,60 \mathrm{~b}$ & $0,05 \mathrm{~m}$ & & $8,80 \mathrm{a}$ \\
\hline $0,4 \mathrm{mg}-1$ & $14,60 \mathrm{a}$ & $0,05 \mathrm{mg}-3$ & 14,40 & & & $g-1$ & $11,20 \mathrm{~b}$ & $0,05 \mathrm{~m}$ & & $7,80 \mathrm{a}$ \\
\hline $0,4 \mathrm{mg}-2$ & $13,20 \mathrm{a}$ & $0,05 \mathrm{mg}-4$ & 17,20 & & & $g-2$ & $8,80 \mathrm{a}$ & $0,05 \mathrm{~m}$ & & $10,20 \mathrm{a}$ \\
\hline $0,4 m g-3$ & $15,00 \mathrm{a}$ & $0,025 \mathrm{mg}-1$ & 11,00 & & & $g-3$ & $11,20 \mathrm{~b}$ & $0,025 \mathrm{r}$ & $g-1$ & $8,20 \mathrm{a}$ \\
\hline $0,4 m g-4$ & $16,40 \mathrm{a}$ & $0,025 \mathrm{mg}-2$ & 13,00 & & & $g-4$ & $11,80 \mathrm{~b}$ & $0,025 \mathrm{r}$ & $g-2$ & $9,20 \mathrm{a}$ \\
\hline $0,3 \mathrm{mg}-1$ & $16,60 \mathrm{a}$ & $0,025 \mathrm{mg}-3$ & 12,40 & & & $g-1$ & $8,80 \mathrm{a}$ & $0,025 \mathrm{r}$ & -3 & $9,40 \mathrm{a}$ \\
\hline $0,3 \mathrm{mg}-2$ & $18,00 \mathrm{a}$ & $0,025 \mathrm{mg}-4$ & 14,40 & & & $g-2$ & $11,80 \mathrm{~b}$ & $0,025 \mathrm{r}$ & $8-4$ & $9,60 \mathrm{a}$ \\
\hline $0,3 \mathrm{mg}-3$ & $15,20 \mathrm{a}$ & $\mathrm{CM}-1$ & 15,40 & & & $g-3$ & $10,60 \mathrm{a}$ & $\mathrm{CM}-$ & & $11,60 \mathrm{~b}$ \\
\hline $0,3 m g-4$ & $17,80 \mathrm{a}$ & $\mathrm{CM}-2$ & 15,20 & & & $g-4$ & $8,20 \mathrm{a}$ & $\mathrm{CM}-$ & & $12,40 \mathrm{~b}$ \\
\hline $0,2 \mathrm{mg}-1$ & $11,20 \mathrm{a}$ & $\mathrm{CM}-3$ & 17,40 & & & $g-1$ & $9,60 \mathrm{a}$ & $\mathrm{CM}-$ & & $12,20 \mathrm{~b}$ \\
\hline $0,2 \mathrm{mg}-2$ & $15,20 \mathrm{a}$ & $\mathrm{CM}-4$ & 12,00 & & & $g-2$ & $10,40 \mathrm{a}$ & $\mathrm{CM}-$ & & $11,40 \mathrm{~b}$ \\
\hline $0,2 \mathrm{mg}-3$ & $13,40 \mathrm{a}$ & $\mathrm{CA}-1$ & 12,60 & & & $g-3$ & $9,20 \mathrm{a}$ & $\mathrm{CA}-$ & & $11,80 \mathrm{~b}$ \\
\hline $0,2 \mathrm{mg}-4$ & $14,80 \mathrm{a}$ & $\mathrm{CA}-2$ & 18,00 & & & $g-4$ & $10,20 \mathrm{a}$ & $\mathrm{CA}-2$ & & $12,80 \mathrm{~b}$ \\
\hline $0,1 \mathrm{mg}-1$ & $16,60 \mathrm{a}$ & $\mathrm{CA}-3$ & 12,80 & & & $g-1$ & $14,00 \mathrm{~b}$ & $\mathrm{CA}-3$ & & $11,00 \mathrm{~b}$ \\
\hline $0,1 \mathrm{mg}-2$ & $18,60 \mathrm{a}$ & $\mathrm{CA}-4$ & 16,40 & & & $g-2$ & $13,00 \mathrm{~b}$ & $\mathrm{CA}-$ & & $13,20 \mathrm{~b}$ \\
\hline
\end{tabular}

$\overline{\mathrm{CA}}=$ controle água; $\mathrm{CM}=$ controle metanol; $\mathrm{FHR}=$ fração hidroalcóolica remanescente.

Scott-Knott (5\% de probabilidade) (Ferreira, 2000). $\mathrm{O}$ tratamento foi considerado efetivo quando todas as repetições estiveram no mesmo grupo de médias.

\section{RESULTADOS E DISCUSSÃO}

Ao analisar as tabelas 1,2 e 3 , verifica-se influência do extrato bruto etanólico $(0,6 \mathrm{mg}, 0,4 \mathrm{mg}$, $0,3 \mathrm{mg}, 0,2 \mathrm{mg}$ e $0,05 \mathrm{mg}$ ) e fração hidroalcóolica remanescente $(0,3 \mathrm{mg})$ de E. velutina na germinação de $L$. sativa, estimulando-a. Influência inibitória das amostras provenientes de E. velutina sob crescimento de Lactuca sativa foi evidenciada com o Teste de Scott-Knott aplicado no ensaio de crescimento, onde a diferença estatística nas médias das leituras do crescimento do hipocótilo pode ser visualizada para os tratamentos 0,6 $\mathrm{mg}$ e $0,4 \mathrm{mg}$ de extrato bruto etanólico; $0,6 \mathrm{mg}, 0,4 \mathrm{mg}$, $0,3 \mathrm{mg}, 0,2 \mathrm{mg}, 0,1 \mathrm{mg}$ e $0,05 \mathrm{mg}$ de fração hexano; 0,6 $\mathrm{mg}$ de fração clorofórmio e $0,2 \mathrm{mg}, 0,05 \mathrm{mg}$ e $0,025 \mathrm{mg}$ de fração hidroalcóolica remanescente.

A germinação não é somente o fenômeno que, em condições adequadas o eixo embrionário prossegue em seu desenvolvimento. Deve-se ter em mente as fases que ocorrem antes da retomada do desenvolvimento, que se iniciam com a colocação da semente em substrato adequado e absorção de umidade. A primeira fase é caracterizada pelo aumento da intensidade respiratória, início da degradação de substâncias de reserva e desdobramento destas em substâncias de menor tamanho para facilitar o transporte. $\mathrm{Na}$ segunda fase ocorre o transporte das substâncias desdobradas, diminuição da absorção de água e crescimento lento da intensidade respiratória. A partir de um teor de umidade (50 a $60 \%$ para as cotiledonares), a semente retorna à intensa absorção de água e respiração, iniciando-se crescimento visível do eixo embrionário e a terceira fase. Substâncias desdobradas na primeira fase e transportadas na segunda fase são reorganizadas em substâncias complexas que, na terceira fase permitem o crescimento do eixo embrionário (Carvalho \& Nakagawa, 1988). O hipocótilo e a radícula são originados a partir do eixo embrionário, parte vital da semente com tecido meristemático em duas extremidades, com condições de crescimento para dois sentidos, o das raízes (radícula) e o do caule (hipocótilo), originando plântula com condições de fixação ao solo e de fotossintetisar substâncias necessárias (Carvalho \& Nakagawa, 1983). Nesta perspectiva, sabe-se que o crescimento é resultado da germinação, portanto, alterações na fase da germinação poderão originar plântulas com dificuldade de crescimento normal, justificando o estímulo da germinação de L. sativa tratada com amostras de E. velutina e posterior inibição do crescimento do hipocótilo.

Estes resultados são indicativos de potencial biotecnológico da espécie, sendo assim, novos ensaios deverão ser conduzidos com as mesmas amostras com propósito de identificar os constituintes químicos responsáveis pelos resultados aqui obtidos. 


\section{REFERÊNCIAS}

Adegas FS, Voli E, Prete CEC 2003. Embebição e germinação de sementes de picão-preto (Bidens pilosa). Planta Daninha 21: 21-25.

Agra MF, Silva KN, Basílio IJLD, França PF, Barbosa-Filho JM 2008. Survey of medicinal plants used in the region Northeast of Brazil. Rev Bras Farmacogn 18: 472-508.

Carvalho NM, Nakagawa J 1983. Sementes: ciência, tecnologia e produção. Campinas: Fundação Cargill.

Carvalho NM, Nakagawa J 1988. Sementes: ciência, tecnologia e produção. Campinas: Fundação Cargill.

Carvalho JLS 2001. Contribuição ao estudo fitoquímico e analítico de Nasturtium officinale R.BR., Brassicaceae. Curitiba. Dissertação de Mestrado - Programa de Pós-Graduação em Ciências Farmacêuticas, Universidade Federal do Paraná.

Chon S-U, Jang H-G, Kim D-K, Kim Y-M, Boo H-O, Kim Y-J 2005. Allelopathic potential in lettuce (Lactuca sativa) plants. Scientia Horticulturae 106: 309-317.

Corrêa MFP, Melo GO, Costa SS 2008. Substâncias de origem vegetal potencialmente úteis na terapia da Asma. Rev Bras Farmacogn 18 (Supl.): 785-797.

Cunha EVL, Dias C, Barbosa-Filho JM, Gray AI 1996. Eryvellutinone, an isoflavone from the stem bark of Erythrina vellutina. Phytochemistry 43: 1371-1373.

De Feo V, De Simone F, Senatore F 2002. Potential allelochemicals from the essential oil of Ruta graveolens. Phytochemistry 61: 573-578.

Dias JFG 2005. Estudo alelopático aplicado de Aster lanceolatus, Willd. Curitiba. Dissertação de Mestrado - Programa de Pós-Graduação em Ciências Farmacêuticas, Universidade Federal do Paraná.

Dias JFG, Círio GM, Miguel MD, Miguel OG 2005 Contribuição ao estudo alelopático de Maytenus ilicifolia Mart. ex Reiss., Celastraceae. Rev Bras Farmacogn 15: 220-223.

Ferreira DF 2000. Sistema de análise de variância de dados balanceados (SISVAR). Pacote computacional. Lavras: UFLA.

Lorenzi H 2002. Árvores brasileiras, manual de identificação e cultivo de plantas arbóreas nativas do Brasil. São Paulo: Instituto Plantarum de Estudos da Flora.

Macías FA, Castellano D, Molinillo JMG 2000. Search for a standard phytotoxic biossay for allelochemicals. Selection of standard target species. J Agric Food Chem 48: 2512-2521.

Maguires JD 1962. Speed of germination - aid in selection and evaluation for seedling emergence and vigor. Crop Sci 2: 176-177.

Malheiros A, Peres MTLP 2001. Alelopatia: interações químicas entre espécies. In: Yunes RA, Calixto JB. Plantas medicinais sob a ótica da química medicinal moderna. Chapecó: Argos, p. 503-523.

Pinto AC, Silva DHS, Bolzani VS, Lopes NP, Epifanio RA 2002. Produtos naturais:atualidade, desafios e perspectivas. Quim Nova 25: 45-61.
Rabelo LA, Agra MF, Cunha EVL, Silva MS, Barbosa-Filho JM 2001. Homohesperetin and phaseollidin from Erythrina velutina. Biochem Syst Ecol 29: 543-544.

Reyes AEL 2008. Trilhas da ESALQ. Árvores úteis. Erythina velutina Wild. [http://www.esalq.usp.br/trilhas/uteis/ ut02.php]. Acesso em 28/08/2008.

Sousa FCF, Melo CTV, Citó MCO, Félix FHC, Vasconcelos SMM, Fonteles MMF, Barbosa-Filho JM, Viana GSB 2008. Plantas medicinais e seus constituintes bioativos: Uma revisão da bioatividade e potenciais benefícios nos distúrbios da ansiedade em modelos animais. Rev Bras Farmacogn 18: 642-654.

Virtuoso S, Davet A, Dias JFG, Cunico MM, Miguel MD, Oliveira AB, Miguel OG 2005. Estudo preliminar da atividade antibacteriana das cascas de Erythrina velutina Willd., Fabaceae (Leguminosae). Rev Bras Farmacogn 15: 137- 142. 